\title{
A Comparative Evaluation for Retention of Maxillary Complete Denture Using Different Commercially Available Denture Adhesives: An In Vivo Study
}

\author{
Samarth K Agarwal ${ }^{1}$, Manish Kumar ${ }^{2}$, Romil Singhal ${ }^{3}$, Akanksha Singh ${ }^{4}$, Kumari Kalpana ${ }^{5}$
}

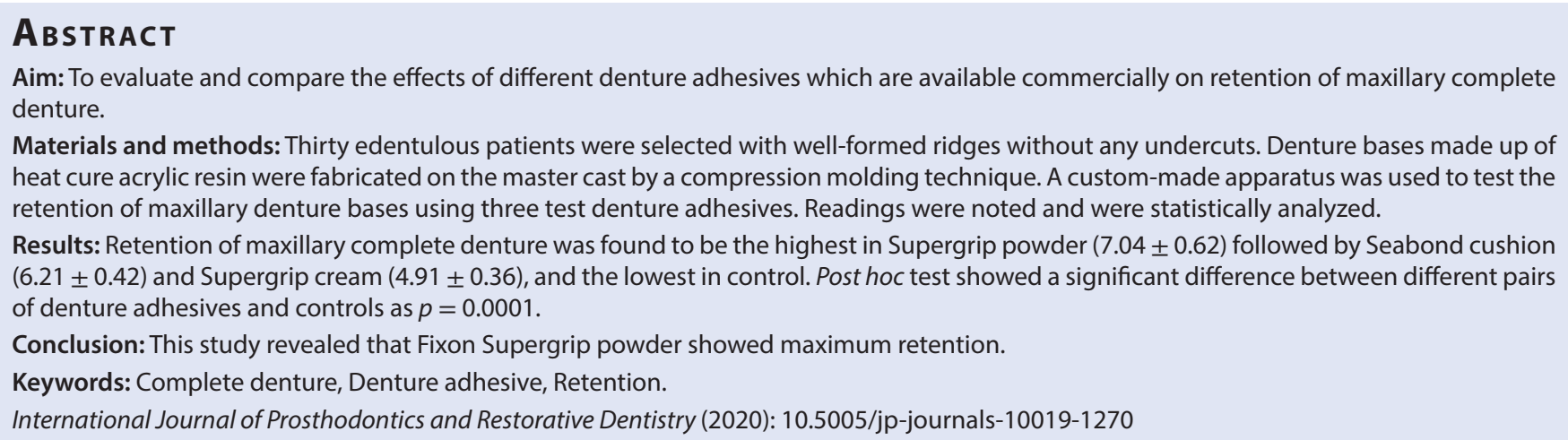

\section{INTRODUCTION}

The goal of prosthetic dentistry has always been the improvement of retention and stability, major properties that determine the performance of a removable prosthesis. Complete denture wearers are often challenged with varying proportions of looseness of their prosthesis and complain of displeasure and/or reduced masticatory function or speech. Edentulous patients treated with complete dentures report of both functional disturbances and psychological problems. ${ }^{1,2}$

According to Zarb et al., the term "denture adhesive" refers to a commercially available, nontoxic, soluble material that is applied to the tissue surface of the denture to enhance retention, stability, and function. ${ }^{3}$

The constituents of most denture adhesives are almost consistent. Stafford et al. have identified the major constituents of adhesives. The ingredients fall into three main groups. ${ }^{4,5}$ The first group consists of those that swell, gel, or dissolve in water and display greater and varying degree of viscosity, (e.g., karaya gum, tragacanth gum, pectin, gelatin, methyl cellulose, hydroxymethyl cellulose, sodium carboxy methyl cellulose, synthetic polymers, such as polyethylene oxide, acrylamide, acetic polyvinyl). The second group consists of those materials that act as antiseptic, antibacterial, and antimycotic agents such as hexachlorophene, sodium tetraborate, sodium borate, and ethanol and the third group consists of those additional materials that serve as fillers, preservatives, plasticizers, flavoring, and wetting agents.

Early adhesives were made from vegetable gums such as acacia, tragacanth, or karya that adsorb water to form a mucilaginous layer between the denture-bearing tissue and the denture base, but they were highly soluble in the mouth making the denture
${ }^{1-5}$ Department of Prosthodontics and Crown and Bridge, Kothiwal Dental College and Research Centre, Moradabad, Uttar Pradesh, India Corresponding Author: Akanksha Singh, Department of Prosthodontics and Crown and Bridge, Kothiwal Dental College and Research Centre, Moradabad, Uttar Pradesh, India, Phone: +91 8411865866, e-mail: singhakanksha2706@gmail.com

How to cite this article: Agarwal SK, Kumar M, Singhal R, et al. A Comparative Evaluation for Retention of Maxillary Complete Denture Using Different Commercially Available Denture Adhesives: An In Vivo Study. Int J Prosthodont Restor Dent 2020;10(2):57-61.

Source of support: Nil

Conflict of interest: None

adhesive useful for only a relatively short period. ${ }^{6}$ Manufacturers are continuously changing the composition of denture adhesives to improve the efficacy of their products. Currently, denture adhesives can be divided into soluble and insoluble groups. The insoluble group comprises of pads and synthetic wafers, whereas the soluble group includes creams, pastes, and powders. ${ }^{6}$ Pads and wafers are very different from creams and powders. The unique feature of pads and synthetic wafers is the inclusion of a fabric carrier impregnated with an adhesive.

In a study of Koksal et al., which showed that (56.3\%) of all dentists used denture adhesives in clinical steps, whereas (41.8\%) dentists recommended to their patients when indicated. ${ }^{7}$

Indications of denture adhesives include trial bases, immediate dentures/transitional dentures, reconstruction or pre-prosthetic surgery, psychological support, compromised anatomic structures, physically/mentally challenged patients, xerostomia, and osseointegrated implants. ${ }^{6}$

() The Author(s). 2020 Open Access This article is distributed under the terms of the Creative Commons Attribution 4.0 International License (https://creativecommons. org/licenses/by-nc/4.0/), which permits unrestricted use, distribution, and non-commercial reproduction in any medium, provided you give appropriate credit to the original author(s) and the source, provide a link to the Creative Commons license, and indicate if changes were made. The Creative Commons Public Domain Dedication waiver (http://creativecommons.org/publicdomain/zero/1.0/) applies to the data made available in this article, unless otherwise stated. 
Contraindications of denture adhesives include patients with open cuts or sores in the mouth, an ill-fitting denture, patients allergic to denture adhesives, and patients with broken dentures, missing flanges, or sectional fractures.

Although denture adhesives have been widely accepted by patients, prosthodontists and dental professionals have been hesitant to advocate these over-the-counter products. To date, the topics of their effectiveness, recommended use, and biocompatibility remain a matter of debate in the dental community. ${ }^{8-10}$

The purpose of this study is to evaluate and compare the effect of different commercially available denture adhesives on retention of maxillary complete denture.

\section{Materials and Methods}

Three commercially available different denture adhesives in nature were selected: Fixon Supergrip powder (ICPA Health Products Itd., Ankleshwar, Mumbai), Fixon Supergrip cream (ICPA Health Products Itd. Ankleshwar, Mumbai) and Seabond denture adhesive seals (Combe Incorporated, White Plains, NY, USA). The study was divided into two groups, the experimental group and control group. No adhesive was used in the control group.

The sample size was 30, which was calculated by power analysis by means of clinical software (post hoc power analysis) for an adequate sample size. This showed 28 samples for the study to have $80 \%$ power with a $95 \%$ confidence interval to detect a minimum clinically. We took a sample size of 30 . Blinding and operator bias was done by the randomized application of adhesives.

Thirty patients aged between 45-75 years having firm mucoperiosteum with no signs of inflammation or flabby tissue covering the edentulous ridge and had a normal temporomandibular joint function, with no history of any allergy to dental materials were selected.

Patients having flat or deep palatal vaults, torus palatinus, uneven ridges, knife-edge ridges, unhealed extraction sockets, any mechanical undercuts or bony exostosis, and poor neuromuscular control were excluded for the study.

After obtaining consent from the patient, a maxillary preliminary impression was made with the impression compound and the primary cast was obtained. Custom tray was fabricated using a T-shaped spacer design. The custom tray was stored in water for 24 hours before border molding. Then the tray was bordermolded by tracing compound sticks and the final impression was made with zinc oxide eugenol impression paste and poured in dental stone to obtain master cast.

\section{Fabrication of Maxillary Denture Base}

On the maxillary cast, $2 \mathrm{~mm}$ thick modeling wax denture base was adapted. A 19 gauge wire along with a $U$ shape loop in the center of the wire to aid in the measurement of retention of the denture base is attached between the second premolar and first molar. The wire was placed equidistant from the reference point (incisive papilla) on both sides.

The denture base was processed with heat cure denture base material using a conventional compression molding technique. The denture base was retrieved from the flask and was finished and polished, and stored in water (Fig. 1).

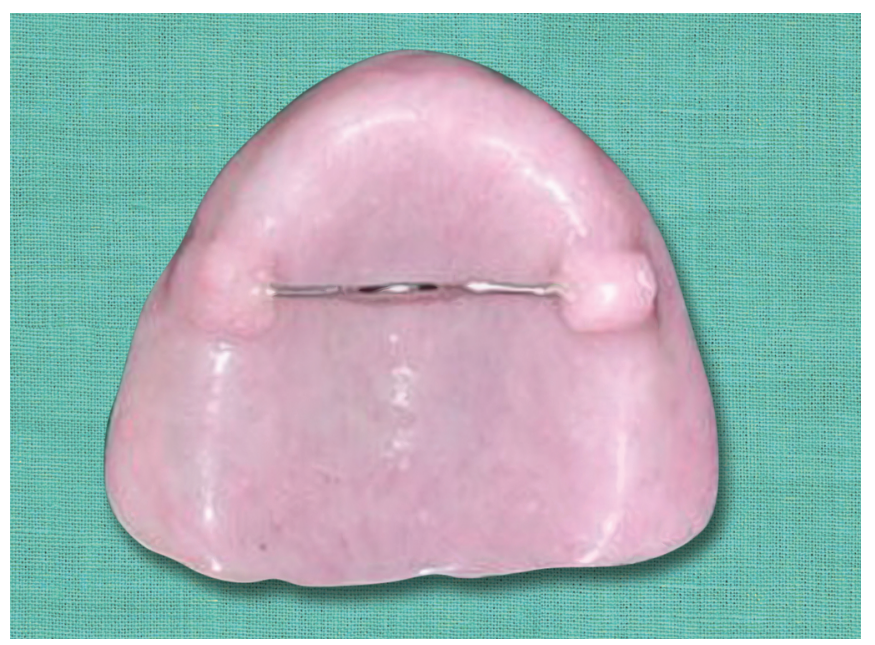

Fig. 1: Denture base with loop

\section{Measurement of Retention of Denture Base}

Retention was measured in terms of vertical force required to dislodge the maxillary denture base from the patient's mouth with the custom-made instrument for the test denture base in every patient.

A custom-made testing instrument was constructed according to Stomberg and Hickey with some modifications to measure the force required to dislodge the dentures in a vertical direction. The testing apparatus had upright support, where two rests were mounted to give support to the subject's head and chin. The forehead rest was so designed that it fitted roughly to the contour of the forehead and was adjusted in an upward and downward motion. The chin rest was designed in the contour of the chin and was adjustable in upward and downward position. An adjustable rod was also attached to the horizontal arm and fastened to the loading receptacle (weighs) in a direct manner.

\section{Testing Procedure}

The patient was seated in an upright position on an adjustable chair in front of the testing apparatus in a comfortable position with his chin rest and forehead leaning solidly against the forehead support. Retention of the maxillary complete denture base was evaluated by applying no denture adhesive and after applying three test denture adhesives randomly, it was tested three times. Retention of denture base with no adhesive (control) and one test adhesive was measured on the first day. The loading receptacle attached to the dislodging rod and loaded with weights until the denture was dislodged from the patient's mouth. The receptacle was weighed and recorded (Fig. 2). After measuring the retention, the denture base was placed in a bowl containing denture cleansing solution, and denture adhesive was removed using a soft brush followed by thoroughly rinsing of denture base under running water for one minute.

On the second day, retention of other denture adhesives was measured. The denture base was thoroughly cleaned and the 2 nd denture adhesive was applied. On the third day, after cleaning, the retention with the third adhesive was measured. After the application of denture adhesives, the base was inserted into the patient's mouth and the patient was asked to close firmly and hold it for two minutes. The retention was measured in the similar manner in all the patients. 
The recorded data were compiled and entered in a spreadsheet (Microsoft Excel) and then exported to the data editor of SPSS Version 20.0 (SPSS Inc., Chicago, Illinois, USA). Data were expressed as mean $\pm S D$. Friedman test was used to compare the retention of maxillary complete denture among different commercially available denture adhesives. The Tukey's honestly significant difference (HSD) post hoc test was used to compare the retention of maxillary complete denture between different commercially available denture adhesives. The $p$ value $<0.05$ was considered significant.

\section{Results}

The Friedman test revealed that the retention of maxillary complete denture was significantly different $(p=0.0001)$ among different commercially available denture adhesives as presented in Table 1.

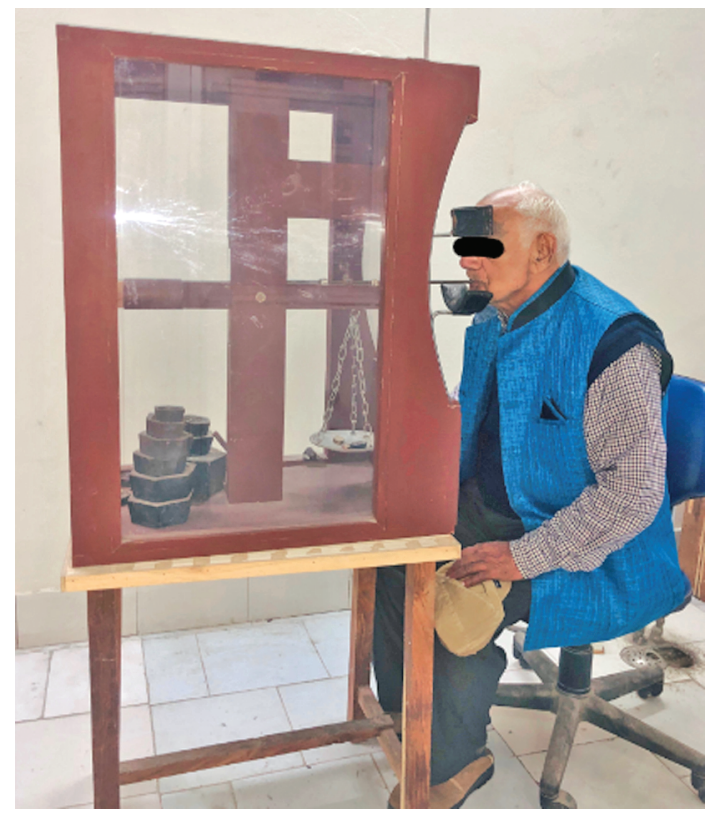

Fig. 2: Testing of measurement for retention of denture base

Table 1: Summarized retention load statistics in different groups

\begin{tabular}{ll}
\hline Groups & $\begin{array}{l}\text { Retention of maxillary complete } \\
\text { denture }(\text { mean } \pm S D)\end{array}$ \\
\hline Control & $2.72 \pm 0.59$ \\
Supergrip powder & $7.04 \pm 0.62$ \\
Supergrip cream & $4.91 \pm 0.36$ \\
Seabond cushions & $6.21 \pm 0.42$ \\
$p$ value & 0.0001 \\
\hline
\end{tabular}

Tukey's HSD post hoc tests showed that the retention of maxillary complete denture had significant $(p=0.0001)$ difference between different pairs of denture adhesives and controls (Table 2).

The retention of maxillary complete denture was found to be the highest in Supergrip powder $(7.04 \pm 0.62)$ followed by Seabond cushions $(6.21 \pm 0.42)$ and Supergrip cream (4.91 \pm 0.36$)$. Retention of maxillary complete denture was the lowest in controls.

\section{Discussion}

Psychological benefit is provided by retention and if a denture can easily be dislodged during speech or mastication, the embarrassment experienced can be mentally traumatic. Retention is affected by various factors such as adhesion, cohesion, interfacial surface tension, mechanical locking into undercuts, peripheral seal, atmospheric pressure, and orofacial musculature.

The use of denture adhesive intensifies the retention through optimizing interfacial forces by (1) increasing the adhesive and cohesive properties, and viscosity of the medium lying between the denture and its basal seat, and (2) getting rid of voids between the denture base and its basal seat.

A special testing apparatus was constructed in accordance with the Stromberg and Hickey ${ }^{11}$ testing device with some modifications. They used an apparatus design, which had both the pulley and lever system to apply forces on the maxillary dentures, but in the present study, the principle of class 3 lever system was used. Here the load travels greater than force travels. Although the amount of effort needed is increased, class 3 levers are useful for increasing the speed at which load is moved. The peripheral seal was broken at once with a greater amount of speed thereby reducing the fast retentive load-bearing capacity of the denture bases. Moreover, the apparatus in the present study was a simple machine with only one lever system and no pulley attachment was there, so forces acting over the pulley were reduced. Intraoral caudal direction forces were selected for dislodgment of denture base as in the patient mouth falls downward, either due to gravitational forces acting on the denture or lack of retentive forces of the denture. ${ }^{12-16}$

In this technique, minimum mouth opening was required so that wire could be placed in the center loop and forces could be transmitted from the anterior end of the dislodging rod to the loop of the denture base. Minimal mouth opening reduces the action of labial and buccal musculature that could easily dislodge the denture bases leading to false reading. ${ }^{12-16}$

Results showed that the retention of maxillary complete denture base was found to be highest in Supergrip powder and it was lowest in controls and was statistically significant ( $p$ value $=$ 0.0001). All the tested denture adhesives have a beneficial impact on improving the retention of maxillary complete denture base with the difference in dislodgment forces from one adhesive to

Table 2: Posthoc comparison tests in pairs of denture adhesives and control

\begin{tabular}{llllll}
\hline & & & & \multicolumn{2}{c}{ 95\% confidence interval } \\
\cline { 4 - 6 } Groups $(I)$ & Groups $(J)$ & Mean difference $(I-J)$ & $p$ value & Lower bound & Upper bound \\
\hline Control & Supergrip powder & 4.31 & 0.0001 & 3.97 & 4.66 \\
& Supergrip cream & 2.19 & 0.0001 & 0.84 & 2.53 \\
Supergrip powder & Seabond cushions & 3.49 & 0.0001 & 3.14 & 3.83 \\
& Supergrip cream & 2.13 & 0.0001 & 1.78 & 2.47 \\
Supergrip cream & Seabond cushions & 0.83 & 0.0001 & 0.48 & 1.18 \\
\hline
\end{tabular}


another. The present study is in the agreement of similar past studies conducted by Manes et al, Pachore and Polyzois et al. ${ }^{17-19}$

Tukey's HSD post hoc tests showed that the retention of maxillary complete denture was found to be statistically significant. For creams and powders, the active and non-active ingredients are essentially same, but the volume of each ingredient may differ; however, the main difference between creams and powders rests with the carrying agent and anti-clumping ingredients. Petrolatum and mineral oil are used in creams but are not present in powders; calcium acetate and silicon dioxide may be used to minimize clumping in powder. ${ }^{6}$

In adhesive cushion, there is a laminated fabric with a wateractivated component impregnated within the fabric's mesh, which becomes sticky upon adsorbing saliva. Webs of the laminate may range from woven napped material to unwoven fiber or web such as light polypropylene scrim or cellulose paper.

The retention value of maxillary complete denture base was found to be highest in Supergrip powder, which was attributed to the fact that denture adhesives are either vegetable gum or synthetic polymer as carboxymethyl cellulose and polyvinyl methyl ether maleate. The contact of carboxymethyl cellulose with saliva leads to the formation of hydrate material, and swells in presence of saliva/water and flows under pressure, thereby getting rid of voids between the denture base and bearing tissue. Hydrate material sticks readily both to the tissue surface of the denture and the mucosal surface of the basal seat, and increases the viscosity of the saliva. These actions markedly increase the retention of complete denture. ${ }^{6,20}$

The higher retentive value of Seabond cushion next to Supergrip powder is attributed to the fact that cushion contains insoluble components which prolong the retention by delaying its dissolution in the saliva. Another advantage of insoluble products over soluble products relates to the cleansibility factor. Because a limited amount of adhesive is incorporated in the fabric carrier, the small amount left in the mouth is easily removed and the pad or synthetic wafer can be readily peeled from the denture base; creams and powders are more difficult to remove because of the relatively large amount of sticky material that remains.

Studies done by Uysal and Lee on adhesive cushions proved to be effective in improving the retention of complete denture wearers. ${ }^{21,22}$

The main components of paste denture adhesive are carboxymethyl cellulose and a polyvinyl group. The action of carboxymethyl cellulose starts instantly after application of the denture adhesive, and with time the long-acting polyvinyl group hydrates and increases adherence and viscosity, also displaying molecular cross-linking resulting in a measurable increase in adhesive behavior. The oily medium in which the active ingredients of the paste are incorporated, delaying the rapid activation of paste denture adhesive, basically increases its duration of action and maintains the higher level of dislodging forces achieved. ${ }^{23}$

From the present study, it is suggested that denture adhesives can be recommended to the patients to improve denture retention irrespective of their form. Though results are favorable, there is further scope to evaluate the effectiveness of denture adhesives in poorly resorbed ridges or patients with poor neuromuscular control.

The uniqueness of the study is the design of apparatus and force application with minimal mouth opening.

There are certain limitations to the present study. Retention of only maxillary well-formed ridges was measured and the retention was measured after two minutes. Therefore, further studies with mandibular ridges with different application time should also be considered to check the effect of different commercially available denture adhesives on the retention of maxillary and mandibular ridges.

\section{Conclusion}

From the present study the following conclusions were drawn:

- The retention of maxillary complete denture was increased with the use of all the three tested denture adhesives.

- The retention of maxillary complete denture was found to be the highest in Supergrip powder followed by Seabond cushions and Supergrip cream.

\section{References}

1. Blomberg S. Psychiatric aspects of patients treated with bridges on osseointegrated fixtures. Swed Dent J Suppl 1985;28:183-192.

2. Albrektsson $T$, Blomberg $S$, Brånemark $A$, et al. Edentulousness-an oral handicap. Patient reactions to treatment with jawbone-anchored prostheses. J Oral Rehabil 1987;14(6):503-511. DOI: 10.1111/j.13652842.1987.tb00746.x.

3. Zarb GA, Bolender $\mathrm{CL}$, Eckert $\mathrm{SE}$, et al. Prosthodontic treatment for edentulous patients: complete dentures and implant-supported prostheses. St Louis. Mosby; 2004. p. 442.

4. Shay K. Denture adhesives.Choosing the right powder and pastes. J Am Dent Assoc 1991;122(1):70-76. DOI: 10.14219/jada. archive.1991.0019.

5. Stafford GO. Denture adhesives-a review of their use and composition. Dent Pract Dent Rec 1970;21(1):17-19.

6. Grasso JE. Denture adhesives. Dent Clin N Am 2004;48(3):721-723. DOI: 10.1016/j.cden.2004.04.002.

7. Koksal T. A survey of dentist' attitude toward denture adhesives. OHDMBSC 2007;6(1):33-39.

8. Agarwal SK. In vitro evaluation of cytotoxicity of denture adhesives. Indian J Dent Res 2011;22(4):526-529. DOI: 10.4103/09709290.90285.

9. Agarwal SK. Comparative evaluation of tissue response to commercially available denture adheives: an in vivo study. J Prosthodont Soc 2012(Suppl 1):159.

10. Kapur KK. A clinical evaluation of denture adhesives. J Prosthet Dent 1967;18(6):550-558. DOI: 10.1016/0022-3913(67)90221-1.

11. Stromberg WR, Hickey JC. Comparison of physiologically and manually formed denture bases. J Prosthet Dent 1965;15(2):213-226. DOI: 10.1016/0022-3913(65)90089-2.

12. Skinner EW. A clinical study of the forces required to dislodge maxillary denture bases of various design. J Am Dent Assoc 1953;47(6):671-680. DOI: 10.14219/jada.archive.1953.0220.

13. Blahova Z. Physical factors in retention of complete denture. J Prosthet Dent 1971;25(3):230-235. DOI: 10.1016/0022-3913(71) 90182-X.

14. Avant WE. A comparison of the retention of complete denture bases having different types of posterior palatal seal. J Prosthet Dent 1973;29(5):484-493. DOI: 10.1016/0022-3913(73)90025-5.

15. Colon A. Analysis of the posterior palatal seal and the palatal form as related to the retention of complete denture. J Prosthet Dent 1982;47(1):23-27. DOI: 10.1016/0022-3913(82)90237-2.

16. Yegin E. Effect of different denture adhesives on retention of complete dentures. An in vivo study. J Adhes Sci Technol 2017;31(18):1-9. DOI: 10.1080/01694243.2017.1296652.

17. Manes JF, Selva EJ, De-Barutell A, et al. Comparison of retention strengths of three complete denture adhesives: an in vivo study. Med Oral Patho Oral Buccal 2011;16(1):132-136. DOI: 10.4317/medoral.16. e132.

18. Polyzois $G$, Lagouvardo $P$, Frangou $M$, et al. Efficacy of denture adhesives in maxillary dentures using gnathodynamometry: a 
comparative study. Odontology 2011;99(2):155-161. DOI: 10.1007/ s10266-011-0003-8.

19. Pachore NJ. Comparative analysis of the effect of three types of denture adhesives on retention of maxillary denture bases. An in vivo study. J Indian Prosthodont Soc 2014;14(4):369-375. DOI: 10.1007/ s13191-013-0334-y.

20. Bajania D, Lagdive S, Shah R. A comparative analysis of the effect of three types of denture adhesives on the retention of maxillary denture bases: an in vivo study. Int J Recent Sci Res 2019;10(7):3399233996.
21. Uysal H. Comparison of four different denture cushion adhesives: a subjective study. JOral Rehabil 1998;25(3):209-213. DOI: 10.1046/j.1365-2842.1998.00220.x.

22. Lee CJ. Comparative adaptation accuracy of acrylic denture bases evaluated by two different methods. Dent Mater J 2010;29(4):411-417. DOI: 10.4012/dmj.2009-105.

23. Chhabra SK, Garg S, Kalra NM. A study to evaluate and compare efficacy of denture adhesives in complete denture patients. Dent J Adv Stud 2018;6(1):7-13. DOI: 10.1055/s-00381671811. 